\title{
Burning Velocities in Catalytically Assisted Self-Propagating High-Temperature Combustion Synthesis Systems
}

\author{
T. KIM and M. S. WOOLDRIDGE* \\ Department of Mechanical Engineering, University of Michigan, 2350 Hayward Street, \\ Ann Arbor, MI 48109-2125, USA
}

\begin{abstract}
The current work presents an experimental study of catalytically assisted self-propagating high-temperature synthesis (SHS) of the tantalum/carbon material system. The effects of controlled amounts of two gas-phase transport agents (carbon dioxide and vaporized iodine) were examined. The gas-phase transport agents resulted in burning velocities 2 to 4 times faster than burning velocities observed in comparable catalytically assisted and unassisted $\mathrm{Ta} / \mathrm{C}$ SHS systems. The gas-phase transport agents clearly augment the combustion synthesis process. The burning velocities of the catalytically assisted, non-melting SHS systems were found to be strong functions of particle size, pressure and the amount of gas-transport agent present. The results are compared with previous studies of both catalytically assisted and unassisted Ta/C SHS, and discussed in the context of proposed transport mechanisms. (C) 2001 by The Combustion Institute
\end{abstract}

\section{INTRODUCTION}

Self-propagating high-temperature combustion synthesis (SHS) is an attractive means to generate non-oxide ceramic materials. SHS systems are self-purifying, require low energy input, and can be used to rapidly generate large quantities of materials (grams per second production rates) [1]. SHS systems can be designed for a wide range of materials $[1,2]$ and, unlike other methods such as temperature-programmed reaction synthesis, SHS systems do not require toxic reactants [2].

Exciting new variations on traditional SHS methods are expanding the range of material systems that can be effectively produced using combustion synthesis and the quality of the materials produced (e.g., increasing reactantsto-products conversion efficiencies). Variations include fluidized-bed combustion synthesis [3], electric-field activated SHS [4], dynamic-compaction SHS [5], and catalytically assisted SHS [6-8]. These variants hold high promise for control of product microstructure and composition, which is critical to enabling combustion synthesis technologies.

In one category of SHS, two solid reactants are mixed, compacted and then ignited. The combustion reaction propagates through the compact yielding a solid product, typically a metal carbide or boride, for example,

*Corresponding author. E-mail: mswool@umich.edu
$\mathrm{Me}(s)+\mathrm{C}(s) \rightarrow \mathrm{MeC}(s)$

where $\mathrm{Me}$ is a transition metal species such as titanium or tantalum, and $\mathrm{C}$ is carbon (graphite, carbon black, etc.). In catalytically assisted SHS, an additional reactant (referred to as a transport agent) is included in the system. The transport agent aids in the transport of the metal or carbon (or boron) reactant species during combustion. For example, metal transport by halogen species is well known [9-12]. At low temperatures (typically $300-800^{\circ} \mathrm{C}[9,10]$ ), a gas-phase metal halide intermediate is formed via

$\operatorname{Me}(s)+A(g) \rightarrow \operatorname{MeA}(g)$

where A is a halogen species such as iodine. The gas-phase metal halide diffuses much more rapidly than the solid or liquid phase species present in the SHS system. At high temperatures (typically $\mathrm{T}>1000^{\circ} \mathrm{C}[9,10]$ ), the metal is re-condensed and the halogen is released. Thus, the halogen species is not consumed during synthesis. The augmentation of the SHS transport processes adds an additional degree of freedom for controlling the combustion system and hence the resulting product material. The transport agent also facilitates synthesis in systems that may not otherwise be capable of sustaining a combustion reaction.

There are few experimental studies of catalytically assisted SHS systems, particularly where the transport agent has been intention- 
ally added to the combustion system [6-8]. Merzhanov et al. [13] examined the effects of trace oxygen-containing species on SHS systems; however, the oxygen containing species were present in uncontrolled amounts as adsorbed impurities in the reactants. In the works by Shtessel and Dorozhevets [6] and by Kostogorov and Dorozhevets [7], solid-phase halogen precursors $\left(\mathrm{C}_{2} \mathrm{~F}_{4}, \mathrm{C}_{6} \mathrm{Cl}_{6}, \mathrm{I}_{2}\right.$, etc. $)$ were mixed with the other solid phase SHS reactants. The results of Dorozhevets and co-workers indicate significant effects on burning velocity, conversion efficiency and other system characteristics; however, interpretation of the results are complicated by evaporation and mixing effects (regarding both mixing of the solid-phase reactants and mixing of the gas-phase species evolved from the halogen precursors).

Transport agents that are initially present in the gas phase (i.e., do not require in situ evaporation) may hold considerable advantages in comparison to transport reactants that are initially in the solid phase. Issues associated with mixing the solid-phase transport agents with the reactant powders are eliminated with gas-phase transport precursors. Depending on the transport agent used, gases may also be more convenient to handle than solid phase precursors, and generally, very little material is required to achieve good product quality with gas-phase precursors [8]. In addition, the in situ evaporation of solid-phase precursors requires that the evolved gases permeate the SHS sample via diffusion at time scales comparable to the combustion reactions, which may be difficult to achieve in some SHS systems.

In an earlier study, we demonstrated the effects of transport agents on the product materials properties (microporosity, composition, microstructure, etc.) of the tantalum/carbon (Ta/C) SHS system using gas-phase transport precursors [8]. The tantalum/carbon SHS system is an ideal system for the study of catalytically assisted SHS because the adiabatic flame temperature (2900 K [4]) is below the melting point temperature for all the species in the system, including the products and intermediates (see Table 1). As a result, diffusion of the gas-phase intermediates is orders of magnitude more rapid than the alternative solid-phase diffusion transport processes. Hence, these non-
TABLE 1

\begin{tabular}{lc}
\multicolumn{2}{c}{ Melting Points for Tantalum/Carbon Species [22] } \\
\hline & Melting point \\
Species & {$[\mathrm{K}]$} \\
\hline $\mathrm{Ta}$ & 3290 \\
$\mathrm{C}$ & 4765 \\
$\mathrm{TaC}$ & 4153 \\
$\mathrm{Ta}_{2} \mathrm{C}$ & 3600 \\
\hline
\end{tabular}

melting systems are particularly sensitive to the presence of transport agents. The current work complements the previous study of product materials properties and presents the effects of transport agents on the combustion characteristics of the $\mathrm{Ta} / \mathrm{C}$ system when gas-phase transport precursors are used. In particular, the effects of the transport agents on the burning velocity as a function of several system parameters are presented. Two gas-phase transport agents were examined: vaporized iodine $\left(\mathrm{I}_{2}\right)$ and carbon dioxide $\left(\mathrm{CO}_{2}\right)$. These transport agents were selected based on anticipated transport reactions, discussed in detail in Kim and Wooldridge [8]. The results of the current work are compared with previous studies of both catalytically assisted and unassisted $\mathrm{Ta} / \mathrm{C}$ SHS. The results are also discussed in the context of proposed transport mechanisms and relationships between the combustion characteristics and the resulting materials properties.

\section{EXPERIMENTAL PROCEDURE}

A sketch of the experimental facility is provided in Fig. 1. Detailed descriptions of the preparation of the $\mathrm{Ta} / \mathrm{C}$ specimens, the experimental apparatus, and the experimental procedure have been provided previously [8]. Briefly, equimolar mixtures of tantalum and carbon (Ta:C $=1: 1)$ were compacted using a mechanical press $(p=49.2 \mathrm{MPa})$ to form cylindrical specimens (1.28 cm diameter, $2.4-3 \mathrm{~cm}$ height). No binding agents were used. The reactant properties are listed in Table 2.

Two type C, D, and/or G thermocouples were used to determine the burning velocity and to monitor the temperature of the sample. Two thermocouple wells $(1.2 \mathrm{~mm}$ diameter, $5.5 \mathrm{~mm}$ depth) were drilled into each specimen perpen- 


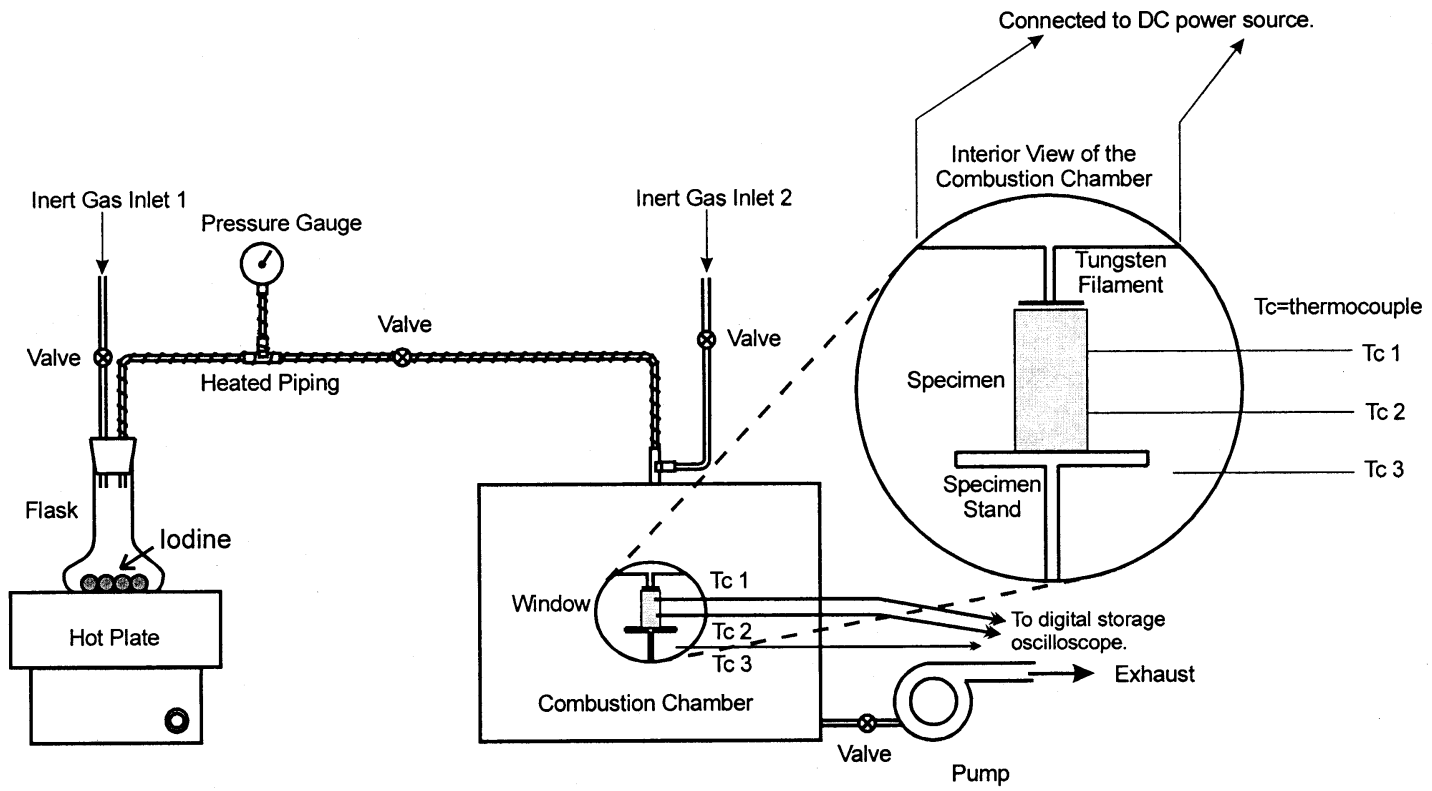

Fig. 1. Experimental schematic of the combustion chamber and gas-handling system. For the carbon dioxide experiments, the iodine source was replaced with a $\mathrm{CO}_{2}$ gas cylinder.

dicular to the cylinder axis at a spacing of $\sim 0.76$ to $1.14 \mathrm{~cm}$. The upper thermocouple was placed $\sim 0.51$ to $1.27 \mathrm{~cm}$ from the top of the specimen and a one mm gap between the ignition source (a tungsten filament) and the specimen was maintained for all experiments. The thermocouple time histories were continuously recorded using a digital oscilloscope. Burning velocities (or reaction front propagation rates) were determined from the temperature profiles and the known spacing between the thermocouples. All flame fronts observed in the current study were planar, and therefore the single point measurements obtained via the thermocouples are considered good indications of the burning velocity.
All experiments were conducted in a cylindrical combustion chamber $(10 \mathrm{~cm}$ diameter, $10 \mathrm{~cm}$ depth). At the start of the experiment, the combustion chamber was sealed, evacuated and purged with argon. The chamber was then filled with argon to the desired partial pressure. For the iodine experiments, $\mathrm{I}_{2}$ was sublimed from crystal powder form and the vapor was directed into the combustion chamber using heated, stainless steel tubing. Iodine vapor was added to the chamber until the desired mole fraction of gas transport agent was achieved. The walls of the combustion chamber were heated for all iodine experiments to prevent significant condensation and loss of the iodine vapor on the chamber walls. (No detectable heating of the

TABLE 2

Reactant Properties

\begin{tabular}{llcr}
\hline Reactant & Manufacturer & Particle size $^{\mathrm{a}}$ & Purity $^{\mathrm{a}}$ \\
\hline Tantalum & Cerac T1000 & $1.88 \mu \mathrm{m}$ & $99.9 \%$ \\
Tantalum & Cerac T2011 & $1.14 \mu \mathrm{m}$ & $99.9 \%$ \\
Carbon black & Fisher C198-500 & $44 \mathrm{~nm}$ & $99 \%$ \\
Iodine & Fisher I35-100 & N/A & $\geq 99.8 \%$ \\
Carbon dioxide & Linde & N/A & $99.9 \%$ \\
Argon & Cryogenic & N/A & $99.998 \%$ \\
\hline
\end{tabular}

\footnotetext{
${ }^{\mathrm{a}}$ As per manufacturer specifications.
} 
TABLE 3

Experimental Conditions Studied in the Current Work

\begin{tabular}{lc}
\hline Parameter & Range \\
\hline Tantalum particle size $^{\mathrm{a}}$ & $1.88 \mu \mathrm{m}, 1.14 \mu \mathrm{m}$ \\
${\text { Pressure }\left(\mathrm{CO}_{2} \text { experiments) }\right.}$ & $0.23-1.60 \mathrm{~atm}$ \\
${\text { Pressure }\left(\mathrm{I}_{2} \text { experiments) }\right.}^{\mathrm{b}}$ & $0.23-1.30 \mathrm{~atm}$ \\
Carbon dioxide mole fraction $^{\mathrm{b}}$ & $40.6-80.0 \%$ \\
Iodine mole fraction $^{\mathrm{b}}$ & $21.4-57.8 \%$ \\
\hline
\end{tabular}

${ }^{a}$ As per manufacturer specifications.

${ }^{b}$ Mole basis of gas-phase species in combustion chamber.

samples occurred prior to ignition in the iodine experiments due to the heating of the chamber walls.) For the $\mathrm{CO}_{2}$ experiments, the iodine source was replaced with a compressed gas cylinder of $\mathrm{CO}_{2}$. The combustion chamber walls were not heated for the $\mathrm{CO}_{2}$ experiments. The mole fraction of gas transport agent in the chamber was determined using the known partial pressures of the gas transport agent and argon. After the desired mole fraction of gas transport agent was obtained, the combustion reaction was initiated by resistively heating the tungsten filament. Power to the filament was immediately discontinued after ignition of the sample. The chamber was immediately purged with argon after each experiment.

\section{RESULTS}

Experiments were carried out using two tantalum reactant particle sizes, a range of mole fractions of gas-transport agent and a range of pressures. The experimental conditions are summarized in Table 3 . The initial temperature $\left(T_{\mathrm{i}}\right)$, the degree of dilution $(\kappa)$, and the sample radius (r) are all known to affect SHS combustion characteristics $[14,15]$ and were kept constant at $\mathrm{T}_{\mathrm{i}}=299 \mathrm{~K}, \kappa=0$, and $r=0.64 \mathrm{~cm}$ throughout the study. As noted above, within the uncertainty of the thermocouple measurements, the initial sample temperature did not change before ignition either due to the heating of the chamber walls or due to the ignition source. Therefore, the initial temperature in the chamber $T_{i}$ is considered equivalent to the initial sample temperature.

The presence of a transport agent in the combustion chamber was critical for ignition of the $\mathrm{Ta} / \mathrm{C}$ compacts. Ignition could not be achieved without a critical minimum quantity of the gas transport agent. A self-sustaining combustion wave was produced only for mole fractions greater than $19 \%$ for $I_{2}$ and greater than $38 \%$ for $\mathrm{CO}_{2}$ (mole basis of gas-phase species). Similarly, Shtessel and Dorozhevets [6] found a minimum threshold value for ignition in their catalytically assisted SHS study of the tantalum subcarbide system using solid phase iodine $(2 \%$ $\mathrm{I}_{2}$, mass basis; Ta: $\mathrm{C}=2: 1$ ).

Results for the burning velocity are presented in Fig. 2 as a function of the concentration of the gas transport agent present in the combustion chamber. There is a clear trend of increasing burning velocity with increasing concentration of gas transport agent for both the $\mathrm{CO}_{2}$ and $\mathrm{I}_{2}$ experiments. However, the iodine data have a much steeper rise with concentration compared to the carbon dioxide data. Note that the specimens prepared with the larger tantalum particles yielded higher burning velocities compared to specimens prepared with the smaller tantalum particles. This trend in particle size is opposite to that observed in unassisted SHS systems (see Discussion below).

In Fig. 3, the data are presented as a function of the mass fraction of the gas transport agent relative to the total mass of participating reactants (i.e., $\mathrm{Ta}(\mathrm{s}), \mathrm{C}(\mathrm{s})$, and $\mathrm{I}_{2}(\mathrm{~g})$ or $\mathrm{CO}_{2}(\mathrm{~g})$ ). Here, the mass of iodine or carbon dioxide available for transport in the compact was approximated as the product of the concentration of the gas transport agent in the chamber and the volume of the compact, that is,

$$
\mathrm{m}_{\text {transport agent }} \cong \mathrm{n}_{\text {transport agent }} \mathrm{V}_{\text {compact }} \text {. }
$$

where $\mathrm{m}$ is the mass of the transport agent $(\mathrm{g})$, $\mathrm{n}$ is the concentration of the transport agent in the combustion chamber $\left(\mathrm{g} / \mathrm{m}^{3}\right)$ and $\mathrm{V}$ is the volume of the compact specimen $\left(\mathrm{m}^{3}\right)$. Relative to the total mass of participating reactants, increases in the mass of $\mathrm{I}_{2}$ or $\mathrm{CO}_{2}$ led to comparable increases in the burning velocity. Therefore, the results indicate that mass fraction is a good basis for comparison of the effects of the amount of gas-phase transport agent on burning velocities.

It is interesting to note that the $\mathrm{CO}_{2}$-assisted experiments yielded burning velocities compa- 


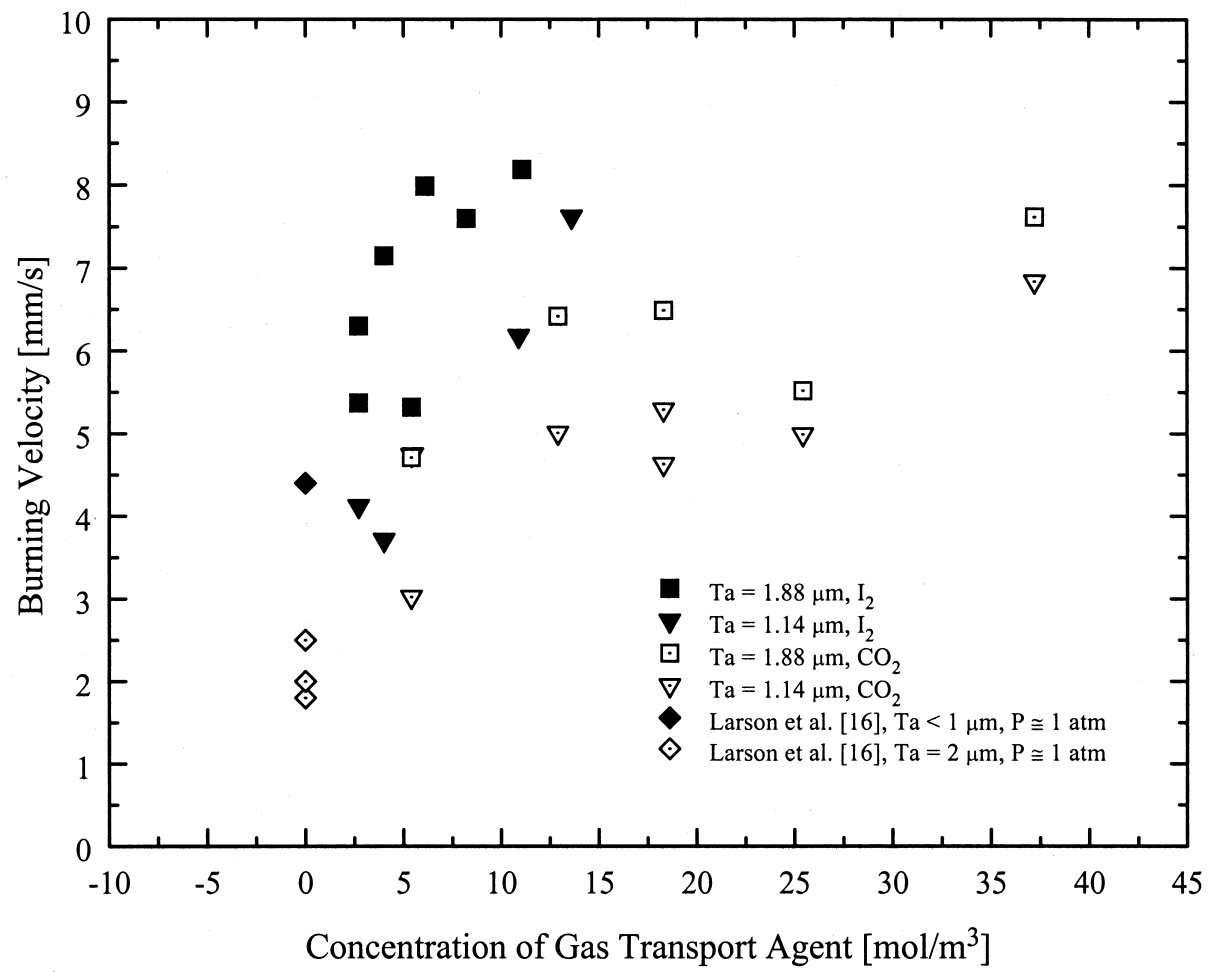

Fig. 2. Experimental results of the effects of gas-phase transport agents on burning velocities for the catalytically assisted $\mathrm{Ta} / \mathrm{C}$ SHS system. The data of Larson et al. [16] are the results of a study of an unassisted Ta/C SHS system.

rable to the $\mathrm{I}_{2}(\mathrm{~g})$-assisted experiments (see Fig. 3 ), given the significant differences between the relevant chemical reactions and transport mechanisms for the two systems. Detailed descriptions of proposed transport and chemical mechanisms are provided in Kim and Wooldridge [8]. In brief, carbon dioxide leads to transport of the carbon to the tantalum, whereas iodine leads to transport of the tantalum to the carbon. Both processes increase contact area between the reactants, which should, and clearly does, result in increased burning velocities in comparison to unassisted systems. The results also indicate that the enhancement provided by $\mathrm{CO}_{2}$ is competitive with that provided by $\mathrm{I}_{2}(\mathrm{~g})$.

Figure 4 shows the effects of pressure on burning velocity for the two transport agents and two tantalum particle sizes. There is a large increase in burning velocity with increasing pressure for all systems studied. Pressure dependence is a clear indication of the significance of the gas-phase reactions and transport mechanisms. Unassisted SHS systems do not typically exhibit pressure dependence [1]. The uncertainties in the velocity measurements are also shown in Fig. 4 as error bars and are predominantly due to the uncertainty in the precise location of the thermocouples in the thermocouple wells. Scatter in the data is primarily attributed to adsorbed impurities in the reactant particles. (The powder reactants were used on an asreceived basis.)

\section{DISCUSSION}

\section{Particle Size Effects}

The results of the catalytically assisted $\mathrm{Ta} / \mathrm{C}$ system are compared with unassisted Ta/C SHS studies in Figs. 2 and 4. Larson et al. [16] examined SHS of $\mathrm{Ta} / \mathrm{C}$ using in situ X-ray diffraction at pressures of approximately one atmosphere. The authors also examined two different size tantalum particles $(\mathrm{Ta}=2 \mu \mathrm{m}$ and $\mathrm{Ta}<1 \mu \mathrm{m}$ ) and found an increase in the 


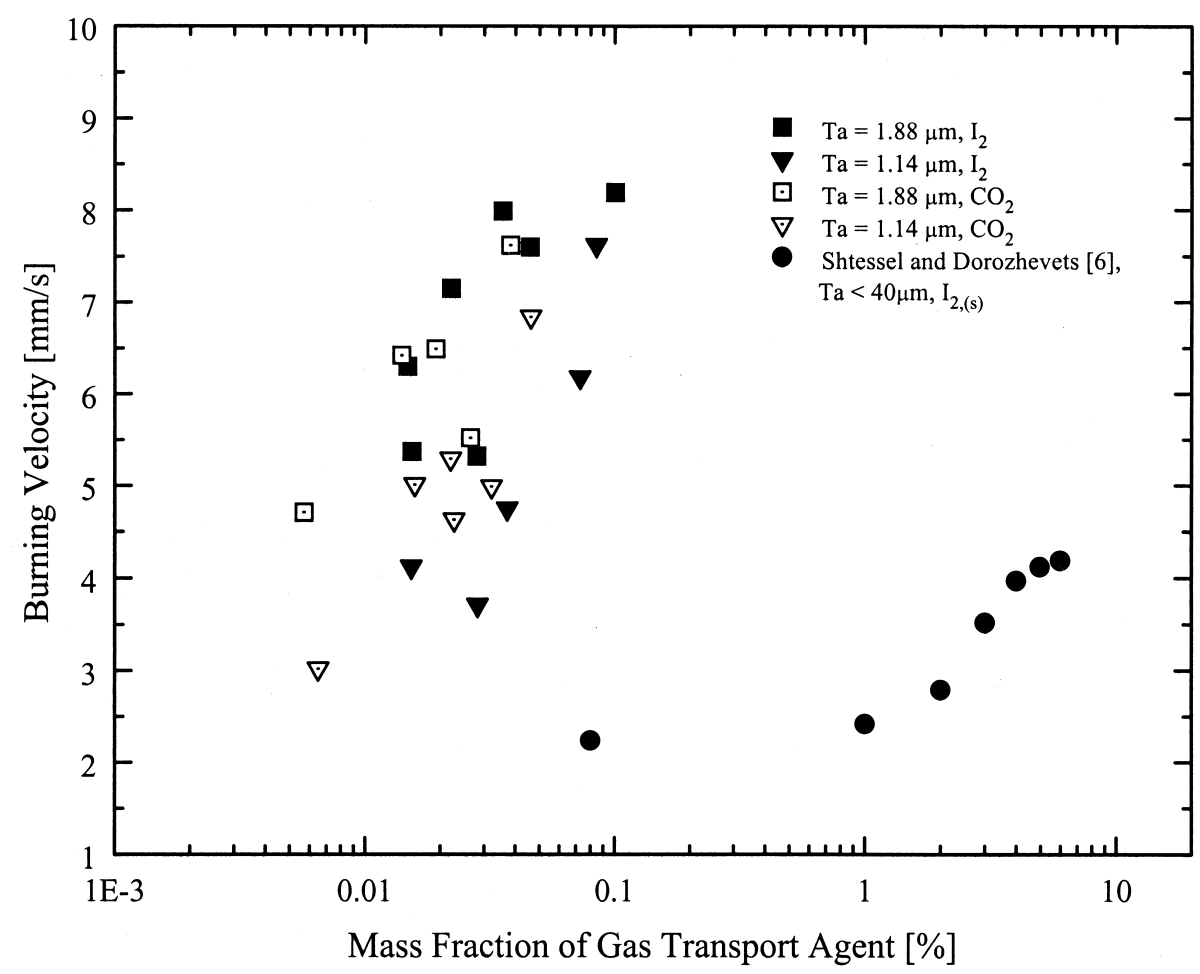

Fig. 3. Experimental results for catalytically assisted Ta/C SHS using $\mathrm{CO}_{2}$, vaporized $\mathrm{I}_{2}$ and solid-phase $\mathrm{I}_{2}$ transport agents. The mass fraction determinations for the current work are based on estimates of the mass of $\mathrm{CO}_{2}\left(\right.$ or $\left.\mathrm{I}_{2}\right)$ available for transport (see text for details). The data of Shtessel and Dorozhevets [6] are the results of a study of catalytically assisted Ta/C SHS where solid-phase iodine was used as the transport agent.

burning velocity with decreasing Ta particle size. Similarly, Shkiro et al. [17] studied particle size effects in unassisted SHS of tantalum carbide $(p=2.25 \mathrm{~atm})$. They found a decrease in burning velocity as fine metal particles $(\mathrm{Ta}=2$ $\mu \mathrm{m})$ were replaced on a mass-fraction basis with coarse metal particles $(\mathrm{Ta} \sim 45 \mu \mathrm{m})$. This inverse relationship between burning velocity and particle size is consistent with other unassisted carbide synthesis studies (e.g., TiC [18]). However, the opposite behavior observed in the current study (i.e., an increase in burning velocity with larger Ta particles) is consistent with an SHS system where gas-phase transport is significant.

All specimens in the current study were compacted using a constant mechanical pressure. As a result, green densities varied slightly between samples prepared using the larger (average green density $=30 \%$ ) and the smaller (average green density $=26 \%$ ) tantalum particles. However, we do not believe the increase in relative density is responsible for the observed trends for two reasons. First, such a small change in relative density does not typically result in such a large change in burning velocity [1] (greater than a factor of 1.5 for many conditions, as seen in Fig. 2). Second, an increase in relative density typically results in a decrease in burning velocity in unassisted $\mathrm{TaC}$ systems $[17,19]$. Instead, the trends in particle size are attributed to the relative porosity of the samples. Estimates for the void volume fraction in the samples indicate greater than $10 \%$ difference between specimens prepared using the large and the small tantalum particles. More accurate determinations of the void volume fractions are not possible with existing particle-packing models due to the complexity of the system (e.g., two polydisperse, agglomerated powders). For the larger tantalum particles, the volume fraction of voids in the compact is smaller, which should result in smaller pores and therefore shorter distances for gas-phase transport to occur. Consequently, 


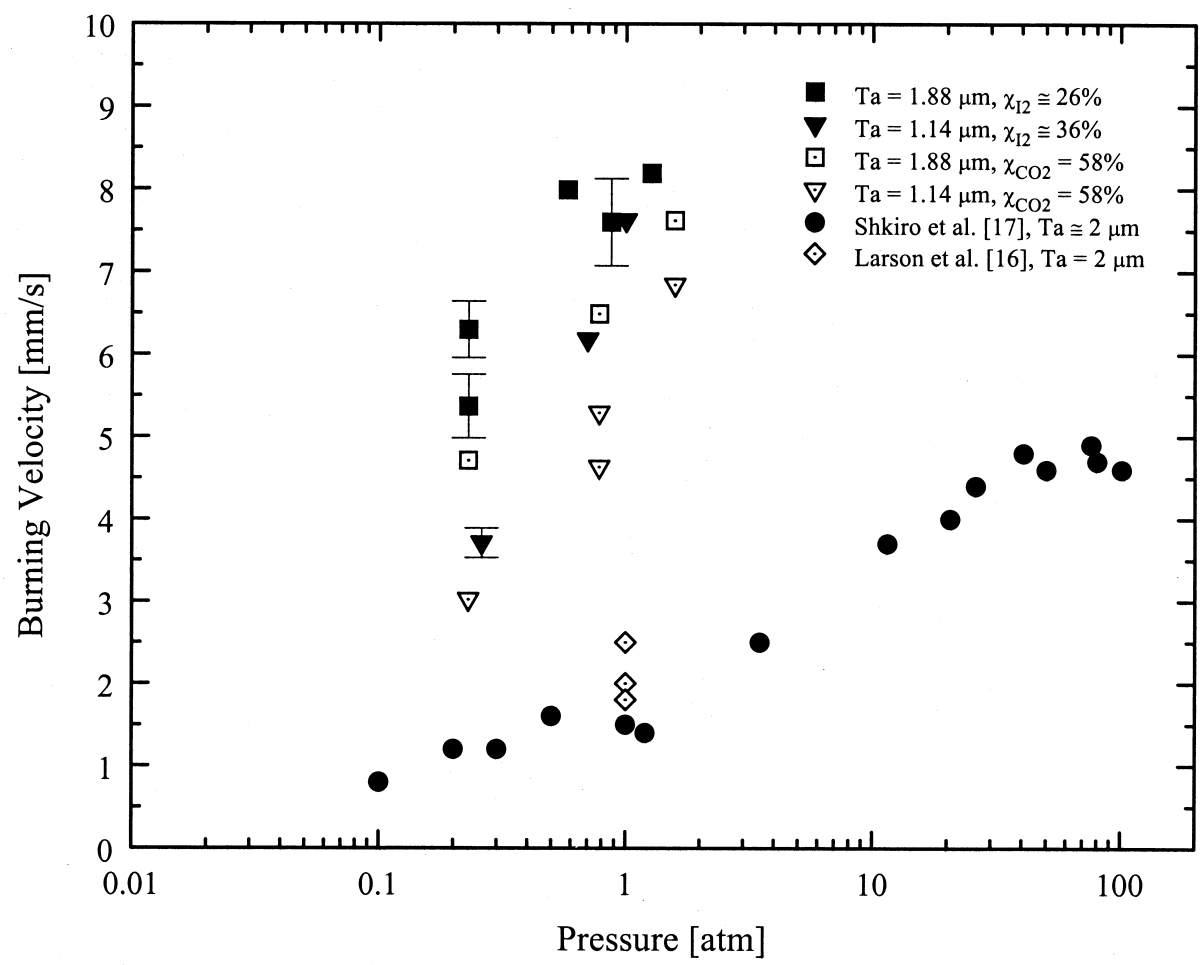

Fig. 4. Experimental results of catalytically assisted and unassisted SHS as a function of pressure for the Ta/C system, where $\chi_{\mathrm{i}}$ is the mole fraction of transport agent $i$ in the combustion chamber (mole basis of gas-phase species). The results of Shkiro et al. [17] and Larson et al. [16] are results of unassisted Ta/C SHS studies. Error bars denote the uncertainty in the determinations of burning velocity in the current work.

diffusion transport by the gas-phase agents should be more rapid in the specimens made using the larger Ta, resulting in higher burning velocities. Note that the diffusion transport of the important gas phase intermediates is very rapid ( $<10 \mu$ s for both transport systems, [10, 13]), particularly in comparison to the characteristic time of the burning velocity. However, as noted previously, the iodine transport system is thermally driven. Consequently, the iodine cannot be re-used multiple times, and only the initial diffusion of the metal halide is critical. Additional discussion of the relevant transport mechanisms and characteristic times is provided in [8].

\section{Pressure Effects}

Pressure effects observed in both catalytically assisted and unassisted [17] Ta/C SHS studies are shown in Fig. 4. The burning velocities associated with the gas-phase transport agents are significantly higher (2-4 times) than the unassisted burning velocities at all conditions studied. Both the unassisted and assisted systems exhibit an increase in burning velocity with increasing pressure; however, the assisted systems have much steeper slopes. Shkiro et al. [19] attribute the pressure dependence to adsorbed impurities in their reactants. At low pressures, gases formed during combustion due to the impurities expand the specimen leading to decreased contact area and therefore reduced burning velocities. This effect (evolution of gas-phase species) is likely augmented in the catalytically assisted SHS systems because of shifts in the transport reactions at higher pressures. For example, tantalum transport via

$$
T a(s)+2 \frac{1}{2} I_{2}(\mathrm{~g}) \rightarrow \mathrm{TaI}_{5}(\mathrm{~g})
$$

is enhanced at higher pressures, when reaction (3) shifts to form additional $\mathrm{TaI}_{5}$. 


\section{Comparisons with Other Catalytically Assisted Ta/C SHS Systems}

Shtessel and Dorozhevets [6] examined the catalytically assisted $\mathrm{Ta} / \mathrm{C}$ system using solid-phase iodine as the precursor material. Their results are included in Fig. 3. The pressure in the combustion chamber was not specified for these experiments. Shtessel and Dorozhevets also noted an increase in the burning velocity with increasing mass fraction of iodine. However, the systems using gas-phase transport agents require significantly less iodine $(\sim$ two orders of magnitude less on a per mass basis) than the systems using solid-phase iodine to achieve the same burning velocities. The results imply that much of the iodine in the solid-phase system is not effectively utilized for transport. This is consistent with the expectation that although there is more iodine present in the solid-phase transport systems, either the gas-phase intermediates have insufficient time to diffuse throughout the specimen, or the gases are restricted to local volumes. Note that the $\mathrm{CO}_{2}$ systems also exhibited higher burning velocities than the solid-phase iodine systems.

\section{Model Comparisons}

The results of the current study were also examined in the context of existing theoretical models for assisted and unassisted SHS systems. Hardt and Phung [20] have proposed a diffusion-limited model for unassisted SHS where $V_{\mathrm{f}}^{2}$ $\propto\left(1 / \mathrm{D}_{\mathrm{p}}^{2}\right) \exp \left(-\mathrm{E}_{\mathrm{A}} / \mathrm{RT}_{\mathrm{c}}\right)$. Here, $\mathrm{V}_{\mathrm{f}}$ is the burning velocity, $\mathrm{D}_{\mathrm{p}}$ is the particle diameter, $\mathrm{E}_{\mathrm{A}}$ is the activation energy for the process and $T_{c}$ is the combustion temperature. Maksimov and Shkadinskii [21] proposed a chemical-kinetics limited model for unassisted SHS where $\mathrm{V}_{\mathrm{f}}^{2} \propto$ $\left(\mathrm{T}_{\mathrm{c}}^{2}\right) \exp \left(-\mathrm{E}_{\mathrm{A}} / \mathrm{RT}_{\mathrm{c}}\right)$. No clear trends were observed consistent with either of the models when the burning velocities of the current work were correlated as $\ln \left(V_{f}^{2}\right)$ as a function of $1 / T_{c}$ or $\ln \left(\mathrm{V}_{\mathrm{f}} / \mathrm{T}_{\mathrm{c}}\right)$ as a function of $1 / \mathrm{T}_{\mathrm{c}}$. In addition, Maksimov and Shkadinskii's model does not include any particle size effects, and the model by Hardt and Phung indicates an increase in burning velocity with smaller particles.

Kostogorov and Dorozhevets [7] developed a model for catalytically assisted SHS using a simplified single-cell configuration. The authors found the burning velocity was a strong function of the parameter $\mathrm{L}$, which is a derived variable that is directly proportional to total pressure, porosity and relative surface area. For most of the modeling conditions examined, the burning velocity increased exponentially with an increase in $\mathrm{L}$, which is consistent with the current experimental results for pressure effects. The model by Kostogorov and Dorozhevets also predicted the burning velocity would approach a constant value at sufficiently high values of $L$. The transition from exponential behavior to the constant value indicated a transition from a deposition-controlled to a kinetically-controlled regime. By comparison, the systems studied in the current work appear to be deposition limited. Note that the porosity defined in the single-cell model is the particle porosity, not the macroscopic void volume fraction. In addition, the Kostogorov and Dorozhevets model does not include particle size effects for the material being transported (e.g., tantalum particle size when iodine is used as the transport agent).

\section{CONCLUSIONS}

The current work presents the first study of catalytically assisted SHS of TaC using controlled amounts of gas-phase transport agents. The gas-phase transport agents resulted in burning velocities 2 to 4 times faster than burning velocities observed in comparable catalytically assisted and unassisted Ta/C SHS systems. The gas-phase transport agents clearly augment the combustion synthesis process. The burning velocities of the catalytically assisted, non-melting SHS systems were found to be strong functions of particle size, pressure and the amount of gas-transport agent present. These system parameters can be used to aid in the design of catalytically assisted combustion synthesis systems. In addition, the experimental results are in good qualitative agreement with trends predicted by the catalytically assisted SHS model of Kostogorov and Dorozhevets [7]. However, existing models do not currently incorporate the physical mechanisms necessary to explain all the trends observed in the catalytically assisted SHS systems studied. 
Ideally, macroscopic SHS characteristics such as the burning velocity can be related to the microscopic characteristics of the final product material. The burning velocity could then serve as a control variable or an indicator of the quality of the product. For many unassisted SHS systems, there appear to be clear qualitative trends where this is the case. For example, qualitative trends indicating a trade-off between burning velocity and percent unreacted carbon are not uncommon (see Shkiro et al. [17, 19], Munir and Anselmi-Tamburini [1], and references therein). A comparison of the results of the current work and the results for the corresponding materials properties (such as percent unreacted carbon, specific surface area, etc. [8]) does not yield such trends. Gas-assisted SHS systems exhibit complex functional relationships between burning velocity and the combustion conditions that complicate extracting simple qualitative relationships. Nevertheless, the transport agents offer the considerable opportunity to extend combustion synthesis methods to new material systems and systems where SHS reactions are otherwise difficult to sustain.

Current and future works include improved modeling efforts to describe catalytically assisted SHS systems, evaluation of additional gas-phase transport agents and material systems, and evaluation of the effects of transport agents on systems where the reactants and/or products can undergo phase change, that is, melting systems.

The authors would like to acknowledge the generous support of the Horace H. Rackham School of Graduate Studies at the University of Michigan.

\section{REFERENCES}

1. Munir, Z. A., and Anselmi-Tamburini, U., Mater Sci. Reports 3:277-365 (1989).
2. Merzhanov, A. G., Combust. Sci. Tech. 98:307-336 (1994).

3. Lee, K. -O., Cohen, J. J., and Brezinsky, K., Proc. Combust. Inst. 28 (2001), in press.

4. Xue, H., and Munir, Z. A., Int. J. SHS 5:229-237 (1996).

5. Wang, L., Wixom, M. R., and Thompson, L. T., J. Mater Sci. 29:534-543 (1994).

6. Shtessel, E. A., and Dorozhevets, I. N., Combust. Expl. Shock Waves 26:52-59 (1990).

7. Kostogorov, E. P., and Dorozhevets, I. N., Intl. J. SHS 1:33-39 (1992).

8. Kim, T., and Wooldridge, M. S., J. Am. Cer. Soc. 2001, in press.

9. Oppermann, H., Solid State Ionics 39:17-25 (1990).

10. Jeffes, J. H. E., and Marples, T. N. R., J. Crys. Growth 17:46-52 (1972).

11. Armas, B., Jeffes, J. H. E., and Hocking, M. G., J. Crys. Growth 44:609-612 (1978).

12. Murray, J. J., Taylor, J. B., and Usner, L., J. Crys. Growth 15:231-239 (1972).

13. Merzhanov, A. G., Rogachev, A. S., Mukas'yan, A. S., Khusid, B. M., Borovinskaya, I. P., and Khina, B. B., J. Eng. Phys. 59:809-816 (1991).

14. Makino, A., and Law, C. K., J. Am. Cer. Soc. 77:778786 (1994).

15. Knyazik, V. A., and Shteinberg, A. S., J. Mater Syn. Proc. 1:85-92 (1993).

16. Larson, E. M., Wong, J., Holt, J. B., Waide, P. A., Nutt, G., Rupp, B., and Terminello, L. J., J. Mater Res. 8:1533-1541 (1993).

17. Shkiro, V. M., Nersisyan, G. A., and Borovinskaya, I. P., Combust. Expl. Shock Waves 14:455-460 (1978).

18. Shkiro, V. M., and Borovinskaya, I. P., in Combustion Processes in Chem Technology and Metallurgy (A. G. Merzhanov, Ed.), Chernogolovka, 1975.

19. Shkiro, V. M., Nersisyan, G. A., Borovinskaya, I. P., Merzhanov, A. G., and Shekhtman, V. Sh., Sov. Powder Met. Metal Cer. 18:227-230 (1979).

20. Hardt, A. P., and Phung, P. V., Combust. Flame 21:77-89 (1973).

21. Maksimov, E. I., and Shkadinskii, K. G., Combust. Explos. Shock Waves 7:392-394 (1971).

22. Lide, D. R., Ed., CRC Handbook of Chemistry and Physics, CRC Press: Boca Raton, 1997.

Received 23 June 2000; revised 16 November 2000; accepted 1 December 2000 